\title{
Câncer em Doença de Crohn: Relato de Caso
}

\author{
Cancer in Crohn's Disease: Case Report
}

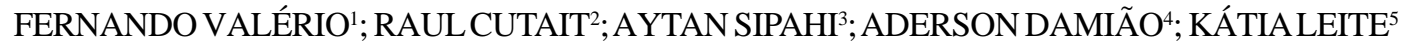

${ }^{1}$ Titular do Colégio Brasileiro de Cirurgia Digestiva; ${ }^{2}$ Professor Associado do Departamento de Cirurgia da Faculdade de Medicina da Universidade de São Paulo; ${ }^{3}$ Chefe da Enfermaria de Gastroenterologia Clínica do Departamento de Gastroenterologia do Hospital das Clínicas e Chefe do Grupo de Intestino da Disciplina de Gastroenterologia Clínica do Hospital das Clínicas; ${ }^{4}$ Professor Assistente da Disciplina de Gastroenterologia Clínica da Faculdade de Medicina da Universidade de São Paulo; ${ }^{5}$ Doutora em Patologia pela Faculdade de Medicina da Universidade de São Paulo, Patologista do Laboratório de Patologia Cirúrgica e Molecular do Hospital Sírio e Libanes.
\end{abstract}

VALÉRIO F; CUTAIT R; SIPAHI A; DAMIÃO A; LEITE K. Câncer em Doença de Crohn: Relato de Caso. Rev bras Coloproct, 2006;26(4): 443-446.

RESUMO: Apesar de se reconhecer que existe um maior risco de câncer em portadores de doença de Crohn (DC), até o presente momento foram descritos menos de 150 casos na literatura mundial. Os principais fatores de risco são a instalação precoce, a longa evolução da doença, a ocorrência de doença fistulosa crônica e a presença de alça exclusa comprometida. Relato de caso. Paciente MCAN, de 53 anos de idade, do sexo feminino, de cor branca, havia sido submetida à ileocolectomia direita aos 17 anos, por obstrução intestinal, decorrente de DC em íleo distal. Há um ano, devido a novo quadro obstrutivo, foi submetida a laparotomia exploradora quando se encontrou massa na região da anastomose ileocólica, caracterizada como adenocarcinoma mucossecretor. Discussão: Na maioria dos casos relatados, o câncer instala-se muitos anos após o início dos sintomas, ocorrendo em cerca de $80 \%$ dos pacientes após 20 anos do diagnóstico de DC. O tumor é habitualmente de crescimento insidioso e leva à obstrução intestinal. Apenas $10 \%$ dos pacientes sobrevivem dois anos livres da doença. $O$ presente caso mostra características identificadas na maioria dos pacientes com DC que desenvolvem neoplasia de intestino delgado: tumor se desenvolvendo muitos anos após a instalação da enfermidade e com comportamento agressivo.

Descritores: Doença de Crohn; câncer.

\section{INTRODUÇÃO}

A relação entre a doença de Crohn (DC) e a presença de lesões malignas ainda não foi bem definida. No entanto, estima-se um maior risco de desenvolvimento de adenocarcinoma de intestino delgado em pacientes com DC do que na população em geral, sendo que, até o presente, foram descritos menos de 150 casos na literatura mundial ${ }^{1,2,3,4,5}$. O número de relatos dessa associação vem crescendo nos últimos anos, provavelmente em decorrência de uma maior preocupação quanto à malignização das lesões granulomatosas da DC e pela melhoria constante dos métodos diagnósticos de imagem ${ }^{6}$, além de evoluções mais longas.

$\mathrm{O}$ primeiro relato associando o câncer à $\mathrm{DC}$ foi de Ginzburg et al $^{7}$ que em 1956 publicaram um caso de adenocarcinoma assestado em segmento de jejuno comprometido por DC em paciente com 30 anos de idade. No Brasil, o primeiro relato de caso de câncer associado à DC foi feito por Mincis et $\mathrm{al}^{8} \mathrm{em} 1982$. Posteriormente, quatro novos casos foram também relatados 9 . No presente trabalho, os autores relatam um caso de adenocarcinoma de intestino delgado em paciente com DC por eles tratado e discutem características clínicas, fatores de risco e a história natural desse tumor em portadores de DC.

\section{RELATO DE CASO}

A paciente M.C.A.N., de 53 anos de idade, do sexo feminino, de cor branca, foi submetida, aos 17 anos de idade, à ileocolectomia direita por obstrução intestinal, decorrente de DC que comprometia o íleo distal. Desde então, apresentava ocasionalmente dores abdominais em cólica. Com 52 anos desenvolveu quadro de obstrução intestinal, tendo sido submetida à

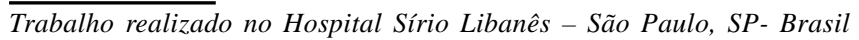

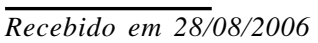

Aceito para publicação em 22/09/2006 
laparotomia exploradora, quando se encontrou massa que comprometia a anastomose ileocólica, a qual foi ressecada com o diagnóstico presuntivo de recidiva da doença. No entanto, o exame anatomopatológico demonstrou a presença de adenocarcinoma mucossecretor, com células em anel de sinete, infiltrando a parede intestinal até o tecido adiposo periférico e com amplo comprometimento linfonodal. Por este motivo, a paciente foi reoperada para ampliação das margens de ressecção, sendo que alguns pontos esbranquiçados em peritôneo visceral, inicialmente interpretados como fibrose ou decorrentes de aderências, eram na realidade implantes metastáticos.

A paciente foi, então, submetida à quimioterapia, mas após alguns meses desenvolveu novo quadro de obstrução intestinal, requerendo ressecção segmentar limitada. Após seis meses, foi reoperada em decorrência de novo episódio de obstrução, desta vez decorrente de importante carcinomatose miliar, tendo sido realizada lise de aderências tumorais e inflamatórias e ressecção de massa metastática em ovário direito. Três meses após apresentou novo quadro obstrutivo, que não se resolveu cirurgicamente, vindo a falecer no período pós-operatório.

\section{DISCUSSÃO}

A DC pode ser encontrada em todo o tubo digestivo, sendo que compromete preferencialmente íleo distal e cólon ( 30 a 55\%), intestino delgado, quase sempre em seu segmento distal (25 a 35\%) e cólon (15 a $25 \%)^{10,11}$. No câncer do intestino delgado, a localização mais comum é o íleo distal (73 a 76\%), ao contrário do que acontece na população geral, em que o duodeno e o jejuno são os segmentos mais acometi$\operatorname{dos}^{1,5,12,13,14,15}$. A ocorrência desse tumor é maior no sexo masculino (3:1), e a faixa etária dos pacientes no momento do diagnóstico é mais precoce ( 45 a 50 anos), quando comparados aos carcinomas do intestino delgado $(65 \text { anos })^{1,2,5,12,15,16}$.

Os fatores de risco para o desenvolvimento de câncer em DC não estão ainda adequadamente definidos, mas se aceita a instalação precoce e a longa evolução da doença inflamatória como fatores associados de risco ${ }^{5,14,16,17}$. Na maioria dos casos relatados, o câncer instala-se muitos anos após o início dos sintomas, ocorrendo em $79 \%$ dos pacientes após 20 anos do início da DC, e em $53 \%$ após 30 anos ${ }^{6,13,17}$. Em nossa paciente tal fato também é observado, já que a pacien- te apresentou tumor após 35 anos do diagnóstico da doença inflamatória. Jess et al ${ }^{18}$, em recente metanálise, mostram que o risco de desenvolvimento de câncer em DC não depende da extensão da doença, mas de sua localização, sendo maior em cólon e reto e menor quando apenas o íleo se encontra comprometido (incidência de 3.4 a $66.7 \%$, média de $27.1 \%$ ). Canavan et al. ${ }^{19}$, por sua vez, também por meio de metanálise, concluem que o risco relativo de câncer em DC de delgado é de 33.2.

Fístulas êntero-cutâneas ou êntero-entéricas são relativamente comuns em pacientes com DC, mas a sua associação com o desenvolvimento tumoral é infreqüente. Sugere-se que o adenocarcinoma possa se desenvolver em sítios de fístulas devido ao estímulo constante de regeneração mucosa ${ }^{20}$. Alguns autores questionam esta afirmação e não descrevem casos semelhantes em seus estudos, acreditando que a doença fistulosa é decorrente da própria lesão tumoral ${ }^{1,16,20}$.

$\mathrm{O}$ risco do desenvolvimento deste tipo de tumor se apresenta aumentado em pacientes portadores de alças intestinais exclusas. De acordo com Greenstein e cols. ${ }^{4}$, um terço dos casos de adenocarcinoma de intestino delgado ocorreram neste tipo de paciente. No entanto, Lashner ${ }^{16}$ questiona essa afirmação, sugerindo que o risco não aumenta simplesmente pela exclusão de alça e, sim, pela presença de alças intestinais com doença ativa por longo tempo, predispondo ao desenvolvimento neoplásico. O fato é que as anuloplastias não parecem predispor ao aparecimento de câncer ${ }^{21}$.

O desenvolvimento tumoral em uma alça intestinal exclusa deve ser suspeitado nos casos com história pregressa longa de DC, em que há uma recidiva tardia dos sintomas, e principalmente quando estes são associados a fístulas ou massas abdominais ${ }^{4}$. Desta forma, nos casos em que os pacientes com DC são submetidos a tratamento cirúrgico e não há contra-indicação de ressecção, esta deve ser realizada como tratamento de escolha ${ }^{22}$.

Os principais sintomas relacionados com o câncer na DC são dor abdominal, obstrução intestinal, massa abdominal palpável, perfuração e sangramento, sendo, em geral, não distinguíveis daqueles da doença em atividade, o que dificulta e até mesmo impede o diagnóstico pré-operatório do tumor ${ }^{12,15,17,22}$. Em cerca de $65 \%$ dos casos, o diagnóstico de câncer é realizado após laparotomia exploradora devido a quadro obstrutivo ${ }^{12,15,17}$. 
$\mathrm{O}$ aspecto mais indicativo de agressividade dos tumores associados à DC relaciona-se com seu tipo histológico, já que em $50 \%$ dos casos estes são mucossecretores e pouco diferenciados ${ }^{23}$. De fato, apenas $10 \%$ dos pacientes sobrevivem dois anos livres da doença ${ }^{1,5,14,15,16,17}$.

Embora a seqüência displasia-carcinoma não tenha sido estabelecida claramente na DC, acredita-se que a maioria dos casos de adenocarcinoma nestes pacientes seja precedida de displasia. Do ponto de vista patológico, a displasia possivelmente constitui uma lesão pré-maligna importante, já que o exame cuidadoso da peça cirúrgica tem permitido comprovar, na maioria das vezes, áreas de tecido displásico adjacentes ao tumor ${ }^{1,5,6,14}$. Este achado anatomopatológico também foi observado no caso por nós descrito, o que está de acordo com as proposições de que o potencial oncogênico da DC se associa à displasia.

Apesar do risco de câncer em DC, não existem orientações definidas quanto à necessidade e periodicidade de exames preventivos quando do envolvimento exclusivo de intestino delgado, à semelhança de consenso para DC de cólon ${ }^{24}$.

\section{CONCLUSÃO}

O caso apresentado mostra características observadas na maioria dos pacientes com doença de Crohn que desenvolvem neoplasia: tumor desenvolvendo-se muitos anos após a instalação da enfermidade, de alta agressividade, com diagnóstico definido pela cirurgia e prognóstico bastante reservado.

\begin{abstract}
Although Crohn's disease (CD) is associated with an increased risk for developing cancer, there are less than 150 cases reported in the world literature. Main risk factors include early onset, a long period of disease, chronic fistulous disease and excluded bowel presence. Case report. MCAN, 53 years old, female, white, has been submitted to a right ileocolectomy at the age of 17, due to bowel obstruction caused by distal ileum CD. A year ago, she presented a new obstructive episode and at laparotomy a tumor mass was identified in the ileocolic anastomosis. Pathology confirmed a mucinous adenocarcinoma. Discussion. The majority of patients with CD who develop intestinal cancer have in common the following items: cancer installs many years after the first symptoms, in around $80 \%$ of the cases after 20 years of the CD diagnosis; bowel obstruction is the main problem; lesions are very aggressive, and only $10 \%$ of the patients remain two years free of the disease.
\end{abstract}

Key words: Crohn disease; cancer.

\section{REFERÊNCIAS}

1. Bernstein D, Rogers A. Malignancy in Crohn's Disease. Am J Gastroenterol, 1996; 91: 434-440.

2. Darke SG, Parks AG, Grogono JL, et al. Adenocarcinoma and Crohn's disease. Br J Surg, 1973; 60: 169-175.

3. Gillen CD, Wilson CA, Walmsley RS, et al. Occult small bowel adenocarcinoma complicating Crohn's disease: a report of three cases. Postgrad Med J, 1995; 71: 172-174.

4. Greenstein AJ, Sachar D, Pucillo A, et al. Cancer in Crohn's disease after divisionary surgery. Am J Surg, 1978; 135: 86-90.

5. Ramos CR, Guillén P, Palomo MJ, et al. Adenocarcinoma de intestino delgado y enfermedad de Crohn. Rev Esp Enf Digest, 1997; 89: 321-324.

6. Korelitz BI. Carcinoma of the intestinal tract in Crohn's disease: results of a survey conducted by the National Foundation for Ileitis and Colitis. Am J Gastroenterol, 1983; 78: 44-46.

7. Ginzburg L, Schneider KM, Dreizin DH, et al. Carcinoma of the jejunum occurring in a case of regional enteritis. Surg, 1956; 39: 347-351.
8. Mincis M, Goldenberg S, Fainzilber S, et al. A associação entre moléstia de Crohn e adenocarcinoma de íleo terminal. Rev Col Bras Cir, 1982; 9: 111-115.

9. Habr-Gama A, Teixeira MG, Vieira MJF, et al. Associação Doença de Crohn e câncer: relato de 4 casos. Rev Bras ColoProct, 1990; 10: 77-82.

10. Gyde SN, Prior P, Macartney JC, et al. Malignancy in Crohn's disease. Gut, 1980; 21: 1024-1029.

11. Kvist N, Jacobsen P, Norgaard HH, et al. Malignancy in Crohn's disease. Scand J Gastroenterol, 1986; 21: 82-86.

12. Fresko D, Lazarus SS, Dotan J, et al. Early presentation of carcinoma of the small bowel in Crohn's disease ("Crohn's carcinoma”). Gastroenterology, 1982; 82: 783-789.

13. Hawker PC, Gyde SN, Thompson H, et al. Adenocarcinoma of the small intestine complicating Crohn's disease. Gut, 1982; 23: 188-193.

14. Marchetti F, Fazio VW, Ozuner G. Adenocarcinoma arising from a strictureplasty site in Crohn's disease. Dis Colon Rectum, 1996; 39: 1315-1321.

15. Senay E, Sachar DB, Keohane M, et al. Small bowel carcinoma in Crohn's disease. Cancer, 1989; 63: 360-363. 
16. Lashner BA. Risk factors for small bowel cancer in Crohn's disease. Digest Dis Sci, 1992; 37: 1179-1184.

17. Ribeiro MB, Greenstein AJ, Heimann TM, et al. Adenocarcinoma of the small intestine in Crohn's disease. Surg Gynecol Obst, 1991; 173: 343-349.

18. Jess T, Gamborg M, Matzen et al. Increased risk of intestinal cancer in Crohn's disease: A meta-analysis of population-based cohort studies. AM J Gastroenterol 2005;100:2724-2729

19. Canavan C, Abrams KR, Mayberry J. Aliment Pharmacol Therf 2006;23:1097-1104

20. Church JM, Weakley FL, Fazio VW, et al. The relationship between fistulas in Crohn's disease and associated carcinoma. Dis Colon Rectum, 1985; 28: 361-366.

21. Fearnhead NS, Chowdhury R, Box B, et al. Long-term followup of strictureplasty for Crohn's disease. Br J Surg 2006;93:475-482
22. Frank JD, Shorey BA. Adenocarcinoma of the small bowel as a complication of Crohn's disease. Gut, 1973; 14: 120124.

23. Bachwich DR, Lichtenstein GR, Traber PG. Cancer in inflamatory bowel disease. Med Clin North Am, 1994; 78: 1399-1412.

24. Itzkowitz S, Present DH. Consensus conference: Colorectal screening and surveillance in inflammatory bowel disease. Inflamm Bowel Did 2005;11:314-321

\section{Endereço para correspondência:}

RAUL CUTAIT

Rua Adma Jafet, 50 - $6^{\circ}$. andar 1.308-50 - São Paulo (SP)

E-mail: rcutait@hsl.org.br 